\title{
Genetic Epidemiology of Type 2 Diabetes in Mexican Mestizos
}

\author{
Eiralí Guadalupe García-Chapa, Evelia Leal-Ugarte, Valeria Peralta-Leal, \\ Jorge Durán-González, and Juan Pablo Meza-Espinoza
}

Facultad de Medicina e Ingeniería en Sistemas Computacionales de Matamoros, Universidad Autónoma de Tamaulipas, Sendero Nacional Km 3, Col. San José, Matamoros, TAMPS, 87349, Mexico

Correspondence should be addressed to Juan Pablo Meza-Espinoza; sirol1073@yahoo.com.mx

Received 14 February 2017; Accepted 18 April 2017; Published 18 May 2017

Academic Editor: Joselyn Rojas

Copyright (c) 2017 Eiralí Guadalupe García-Chapa et al. This is an open access article distributed under the Creative Commons Attribution License, which permits unrestricted use, distribution, and reproduction in any medium, provided the original work is properly cited.

\begin{abstract}
There are currently about 415 million people with diabetes worldwide, a figure likely to increase to 642 million by 2040 . In 2015 , Mexico was the second Latin American country and sixth in the world in prevalence of this disorder with nearly 11.5 million of patients. Type 2 diabetes (T2D) is the main kind of diabetes and its etiology is complex with environmental and genetic factors involved. Indeed, polymorphisms in several genes have been associated with this disease worldwide. To estimate the genetic epidemiology of T2D in Mexican mestizos a systematic bibliographic search of published articles through PubMed, Scopus, Google Scholar, and Web of Science was conducted. Just case-control studies of candidate genes about T2D in Mexican mestizo inhabitants were included. Nineteen studies that met the inclusion criteria were found. In total, 68 polymorphisms of 41 genes were assessed; 26 of them were associated with T2D risk, which were located in $A B C A 1, A D R B 3, C A P N 10, C D C 123 / C A M K 1 D, C D K A L 1, C D K N 2 A / 2 B$, CRP, ELMO1, FTO, HHEX, IGF2BP2, IRS1, JAZF1, KCNQ1, LOC387761, LTA, NXPH1, SIRT1, SLC30A8, TCF7L2, and TNF- $\alpha$ genes. Overall, 21 of the 41 analyzed genes were associated with T2D in Mexican mestizos. Such a genetic heterogeneity compares with findings in other ethnic groups.
\end{abstract}

\section{Introduction}

Type 2 diabetes (T2D) is a metabolic disorder characterized by impaired glucose uptake in muscle and fat, altered glucose-induced insulin secretion, and increased hepatic glucose production, which lead to hyperglycemia. It is the most common type of diabetes and generally occurs in adults [1]. According to the International Diabetes Federation there are currently around 415 million people with diabetes worldwide, a figure likely to increase to 642 million by 2040 [2]. This disorder accounts for high morbidity and mortality due to complications like renal failure, blindness, amputations, cardiovascular disease, and cerebrovascular events [1]. In 2015 there were approximately 5.0 million deaths by diabetes worldwide [2]. With about 7.3 million patients in 2010 [3], our country was second in Latin America and tenth in the world in prevalence of this disorder [4]. Five years later, the number of diabetic patients was estimated to be 11.5 million and our country ranked sixth in the world [2]. In 2011 most frequent morbidities by T2D were renal failure $(24.2 \%)$ and peripheral circulatory complications (17.3\%), and the mortality rate was 70/100,000 inhabitants (http://fmdiabetes.org/wp-content/uploads/2014/11/diabetes2013INEGI.pdf). The complex etiology of T2D includes factors that influence the risk and evolution of the disease, such as ethnicity, poor alimentation, sedentary lifestyle, obesity, dyslipidemia, and family history $[1,5]$. Regarding genetics, worldwide researches have shown association of this disease with numerous allelic variants of nearly 80 candidate genes [6]. The aim of this study is to carry out a literature review about genetic researches conducted in Mexican mestizos for a better understanding of the genetic epidemiology of T2D in our population.

\section{Methods}

A systematic search was done through PubMed, Scopus, Google Scholar, and Web of Science for genetic studies conducted in Mexican mestizo inhabitants with T2D. Key words derived from the phrase "Genetic polymorphisms associated 
with Diabetes Mellitus type 2 in Mexico, Mexican patients and/or Mexican mestizo" were used. Related terms such as "variants", "alleles", and "SNP associated with diabetes, T2DM, or T2D” complemented our search. Just case-control studies of candidate genes performed in Mexican mestizos resident in the country were included. Researches conducted in Mexican native populations were excluded, as well as those done in patients with metabolic syndrome. In surveys that included both patients with metabolic syndrome and patients with T2D, only cases with T2D were registered. Although in the selected studies different models of genotype analyses were used (recessive, dominant, or codominant), solely comparisons between allele frequencies were considered in our review. In studies without described odds ratio (OR), unadjusted OR were estimated from the reported allele or genotype frequencies. Allele comparisons were performed by $2 \times 2$ contingency tables [Yates' correction chisquare test (http://vassarstats.net/odds $2 \times 2 . h t m l$ )] and genotypes were contrasted by chi-square test (https://ihg.gsf.de/ cgi-bin/hw/hwa2.pl). In both comparisons, OR were estimated using 95\% confidence intervals (95\% CI). A $p \leq 0.05$ defined a significant association.

Whenever a polymorphism was analyzed in different studies, data were combined and unadjusted OR for alleles were calculated using a $2 \times 2$ contingency table (Yates' correction chi-square test). However, studies with suspicion of overlapping of patients were not included in this analysis.

\section{Results}

In total, 19 case-control studies on the possible association of genetic polymorphisms with T2D in Mexican mestizos resident in the country were included [7-25]. Altogether, 68 polymorphisms of 41 genes were assessed (Table 1). Of them, 25 were associated with an increased risk for T2D and they were located in 20 genes, namely, $A B C A 1, A D R B 3, C A P N 10$, CDC123/CAMK1D, CDKN2A/2B, CRP, ELMO1, FTO, HHEX, IGF2BP2, IRS1, JAZF1, KCNQ1, LOC387761, LTA, NXPH1, SIRT1, SLC30A8, TCF7L2, and TNF- $\alpha$. Among the variants that showed association there were $4 / 20$ amino acid substitutions, 13/30 intronic sites, $6 / 10$ of promoter region or $5^{\prime}$-flanking region or upstream of gene, $1 / 2$ intergenic regions, and $2 / 6$ of $3^{\prime}$-untranslated or $3^{\prime}$-flanking region of gene. On the other hand, 12 polymorphisms were analyzed by different authors, and concordance was observed in most of them, except for rs3842570 (CAPN10) [11, 13, 14], rs13266634 (SLC30A4) [8, 17], rs7903146 (TCF7L2) [8, $10,12,22]$, and $\operatorname{rs} 1800629(T N F-\alpha)[20,24,25]$. Eleven of these polymorphisms were pooled and analyzed as shown in Table 2. Note that rs4994 (ADRB3) was discarded of this analysis (suspicion of overlap of $[9,10]$ ). Similarly, data by Cruz et al. [10] for rs7903146 and rs12255372 of TCF7L2 were not considered (possible overlapping with the study by Martínez-Gómez et al. [12]). Thus, the 3R allele of rs3842570, which was associated with T2D in a small sample, did not seemingly confer susceptibility to the disease; in contrast, the C allele of rs7754840 (CDKAL1), which evidenced no risk in independent studies, showed association with T2D. Including this allele, a total of 26 polymorphisms and 21 genes were associated with T2D in Mexican mestizos.

\section{Discussion}

This review about genetics of T2D in Mexican mestizo subjects shows that 26 polymorphisms distributed in 21 genes are associated with this disease, so T2D has a high heterogeneity in our population, the same as that in other ethnic groups. Therefore, in some individuals alleles of certain genes are involved, while in others subjects are implicated variants of different genes. A previous conclusion that T2D in Mexican mestizos is genetically homogeneous was based on an analysis of three genetic markers [26] and here appears untenable. Though the Mexican mestizo population has a European genetic ancestry near 30\% [27], not all the alleles conferring diabetes risk in Europeans are associated with T2D in our population [8]. These variations could be related to genetic background, differences in clinical classifications, sample size, selection and analysis criteria, and environmental factors such as obesity, lifestyle, and diet. On the other hand, researches in several ethnic groups have shown association of T2D with genes not yet analyzed in Mexican population $[5,6,28-32]$. It would be important to carry out the analysis of such genes to determine whether these variants are also associated with T2D in Mexican patients and increase the knowledge about the genetic epidemiology of this disorder in our country.

Regarding Mexican studies, an increased risk was detected when analysis was performed adjusting covariates. For instance, Cruz et al. observed an additive effect in the T2D risk when they considered variables such as age, education, sex, body mass index, and ancestry [10]. Gamboa-Meléndez et al. reported association with T2D for the polymorphisms rs7923837 (HHEX), rs4402960 (IGF2BP2), and rs2237892 (KCNQ1) only when ancestry was adjusted [8]. For the polymorphisms rs864745 (JAZF1) and rs757705 (NXPH1), the analysis stratified by ancestry did not show significant differences, whereas an association was observed in the comparison without such an adjustment. In addition, they found association for rs7903146 (TCF7L2) and rs7754840 (CDKAL1) just in early-onset T2D [OR $=1.39(1.04-1.85), p=$ $0.024]$ and in nonobese T2D patients [OR $=1.25(1.06-1.49)$, $p=0.009]$, respectively. Another study found a lower OR when the analysis was adjusted by sex, body mass index, and family history of T2D for three polymorphisms of IRS1 in a dominant model [19].

The reported association of rs3842570 (CAPN10) [14], rs909253 (LTA) [20], and rs1800629 (TNF- $\alpha$ ) [24] with T2D should be interpreted with caution given the small sample sizes and poor statistical power. With respect to the rs1345365 polymorphism (ELMO1), the authors reported a protector effect for the A allele [OR $=0.65$ (0.55-0.78), $p<0.001]$ [16]. But in our analysis we took as reference the A allele, as it is the most common; thus, the $\mathrm{G}$ allele showed association with T2D [OR $=1.37$ (1.02 to 1.84$), p=0.035$ ].

Since T2D is a complex disorder and several genes are implicated in its etiology and evolution, the identification 


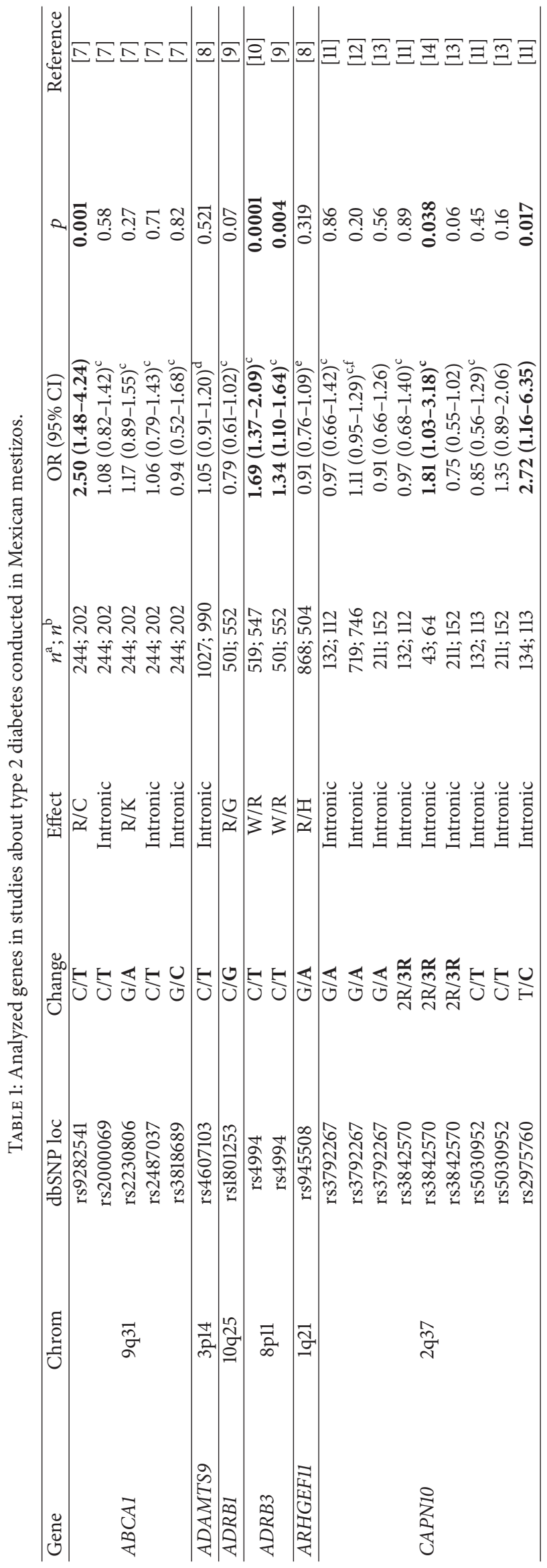



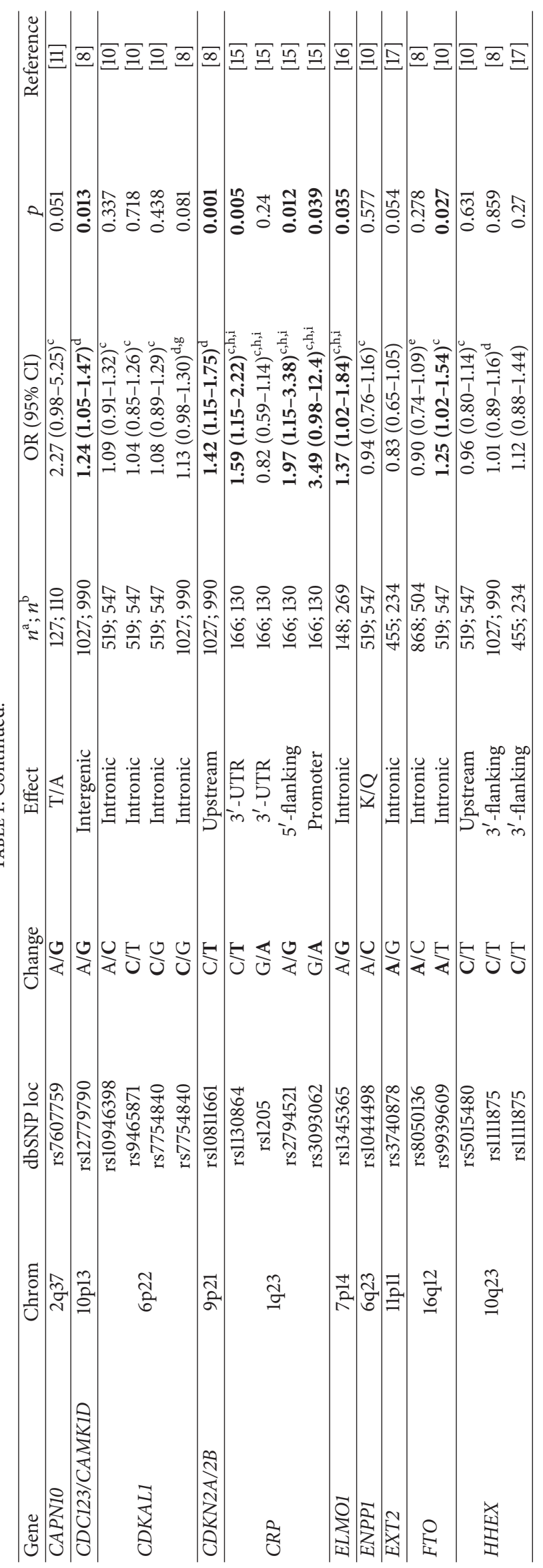


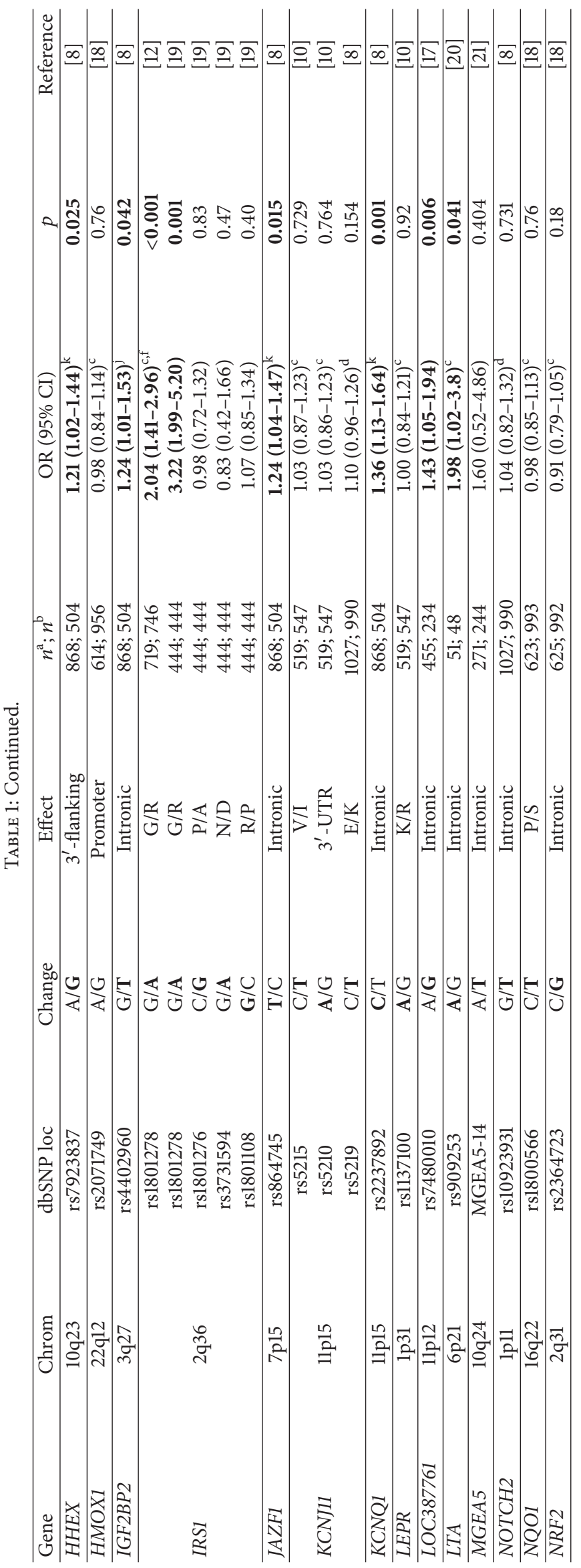




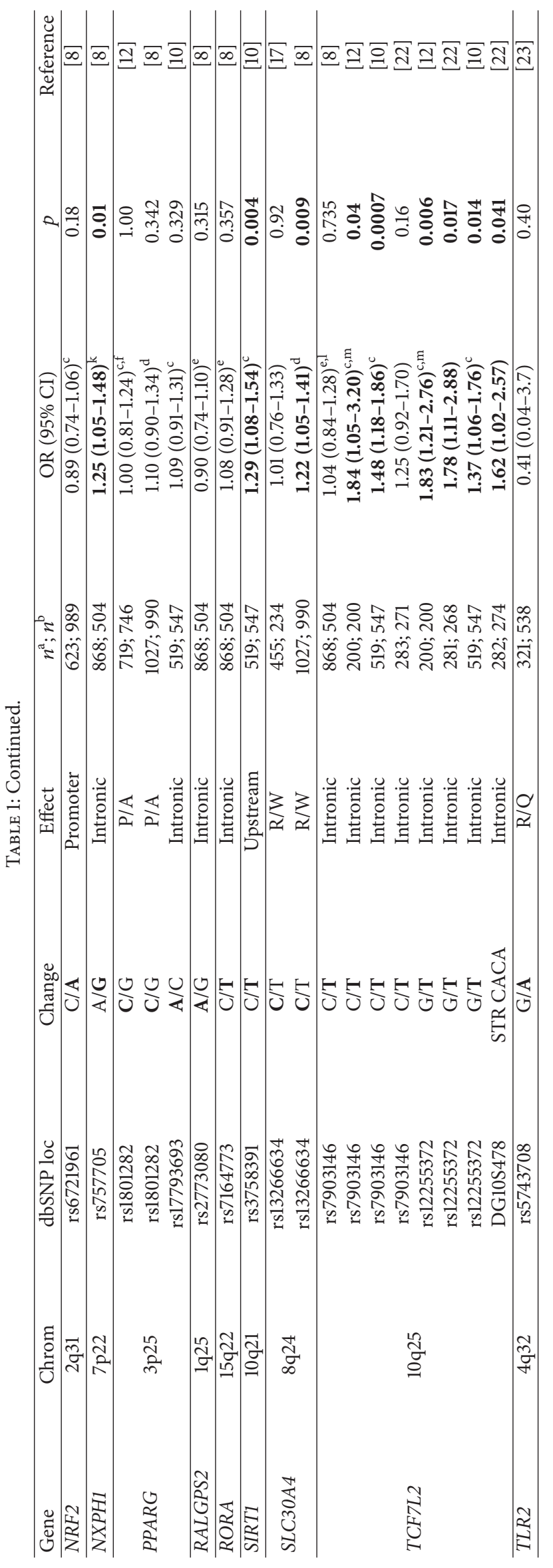




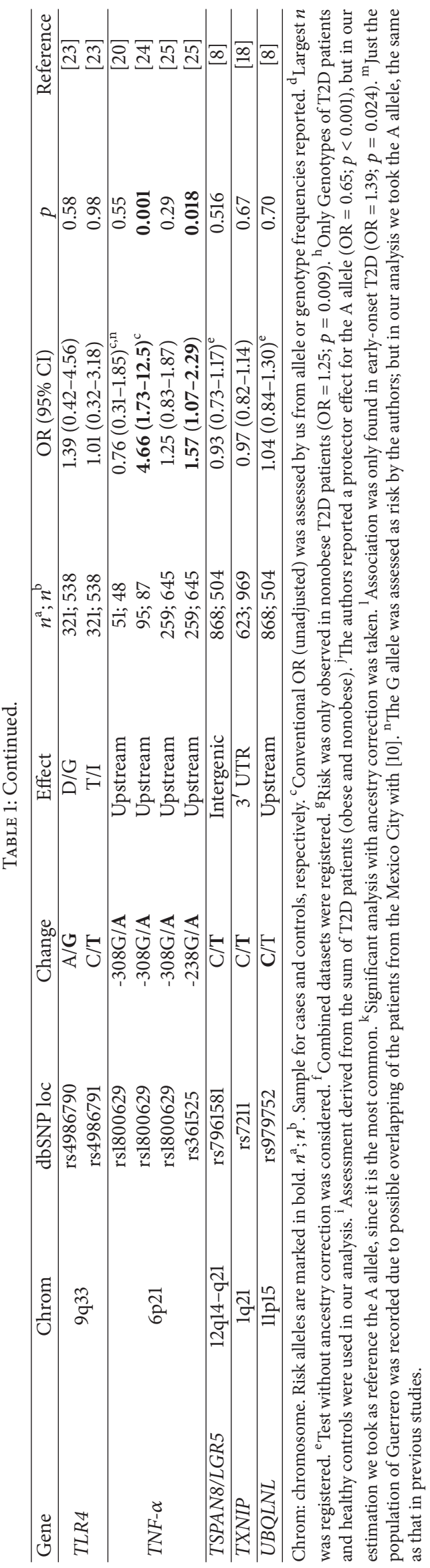




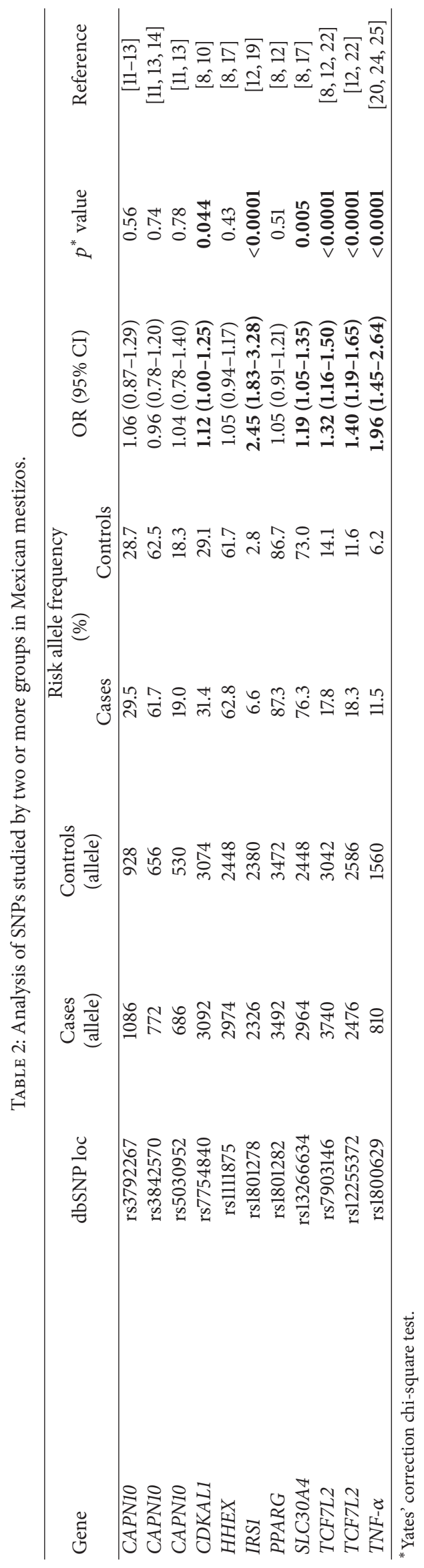


of risk alleles could be useful, because if the involved genes and their function are known, it is more probable to achieve prevention, treatment, prognosis, and/or cure of the disease. Complications could also be prevented or treated better [29, 33]. However, published studies demonstrate that genetic screening for the prediction of T2D in high risk subjects is currently of little value in clinical practice. Actually, genetic risks are difficult to calculate because several alleles could contribute to an additive effect to T2D susceptibility [34], not to mention the diverse environmental factors involved. Although some of these genes are implicated in the glucose and fat metabolism, $\beta$-cell function, and sensitivity and secretion of insulin $[29,35]$, how some of their variants increase the T2D risk remains to be elucidated [29]. Anyway, it is fundamental to analyze the genetic epidemiology of this disease in each population because of the underlying differences in genetic background and lifestyle among ethnic groups. So, it is possible that polymorphisms associated with T2D in some races do not show association in others. Genome-wide association studies will ultimately precise the genetic landscape.

\section{Conflicts of Interest}

The authors declare no conflicts of interest concerning the publication of this paper.

\section{Authors' Contributions}

Eiralí Guadalupe García-Chapa and Juan Pablo MezaEspinoza contributed equally to this work. All the authors participated in drafting and review of this paper.

\section{Acknowledgments}

The authors thank Dr. Horacio Rivera for his support and critical review of the manuscript.

\section{References}

[1] American Diabetes Association, "Standards of medical care in diabetes-2013," Diabetes Care, vol. 36, supplement 1, pp. S11S66, 2013.

[2] International Diabetes Federation, "IDF diabetes atlas," in International Diabetes Federation, pp. 1-144, Brussels, Belgium, 7th edition, 2015, http://www.diabetesatlas.org.

[3] S. Villalpando, V. de la Cruz, R. Rojas et al., "Prevalence and distribution of type 2 diabetes mellitus in Mexican adult population. A probabilistic survey," Salud Pública de México, vol. 52, supplement 1, pp. s19-s26, 2010.

[4] J. E. Shaw, R. A. Sicree, and P. Z. Zimmet, "Global estimates of the prevalence of diabetes for 2010 and 2030," Diabetes Research and Clinical Practice, vol. 87, no. 1, pp. 4-14, 2010.

[5] V. Lyssenko, A. Jonsson, P. Almgren et al., "Clinical risk factors, DNA variants, and the development of type 2 diabetes," New England Journal of Medicine, vol. 359, no. 21, pp. 2220-2232, 2008.

[6] A. Stančáková and M. Laakso, "Genetics of type 2 diabetes," Endocrine Development, vol. 31, pp. 203-220, 2016.
[7] M. T. Villarreal-Molina, M. T. Flores-Dorantes, O. ArellanoCampos et al., "Association of the ATP-binding cassette transporter A1 R230C variant with early-onset type 2 diabetes in a Mexican population," Diabetes, vol. 57, no. 2, pp. 509-513, 2008.

[8] M. A. Gamboa-Meléndez, A. Huerta-Chagoya, H. MorenoMacías et al., "Contribution of common genetic variation to the risk of type 2 diabetes in the Mexican Mestizo population," Diabetes, vol. 61, no. 12, pp. 3314-3321, 2012.

[9] A. I. Burguete-García, G. A. Martínez-Nava, A. ValladaresSalgado et al., "Association of $\beta 1$ and $\beta 3$ adrenergic receptors gene polymorphisms with insulin resistance and high lipid profiles related to type 2 diabetes and metabolic syndrome," Nutrición Hospitalaria, vol. 29, no. 6, pp. 1327-1334, 2014.

[10] M. Cruz, A. Valladares-Salgado, J. García-Mena et al., "Candidate gene association study conditioning on individual ancestry in patients with type 2 diabetes and metabolic syndrome from Mexico City," Diabetes Metabolism Research and Reviews, vol. 26, no. 4, pp. 261-270, 2010.

[11] L. Del Bosque-Plata, C. A. Aguilar-Salinas, M. T. Tusié-Luna et al., "Association of the calpain-10 gene with type 2 diabetes mellitus in a Mexican population," Molecular Genetics and Metabolism, vol. 81, no. 2, pp. 122-126, 2004.

[12] L. E. Martínez-Gómez, M. Cruz, G. A. Martínez-Nava et al., “A replication study of the IRS1, CAPN10, TCF7L2, and PPARG gene polymorphisms associated with type 2 diabetes in two different populations of Mexico," Annals of Human Genetics, vol. 75, no. 5, pp. 612-620, 2011.

[13] V. J. Picos-Cárdenas, E. Sáinz-González, A. Miliar-García et al., "Calpain-10 gene polymorphisms and risk of type 2 diabetes mellitus in Mexican mestizos," Genetics and Molecular Research, vol. 14, no. 1, pp. 2205-2215, 2015.

[14] Y. Loya-Méndez, G. Reyes Leal, A. Sánchez González, V. Portillo Reyes, D. Reyes Ruvalcaba, and G. Bojorquez Rangel, "SNP-19 genotypic variants of CAPN10 gene and its relation to diabetes mellitus type 2 in a population of Ciudad Juarez," Nutrición Hospitalaria, vol. 31, no. 2, pp. 744-750, 2015.

[15] A. Martínez-Calleja, I. Quiróz-Vargas, I. Parra-Rojas et al., "Haplotypes in the CRP gene associated with increased BMI and levels of CRP in subjects with type 2 diabetes or obesity from Southwestern Mexico." Experimental Diabetes Research, vol. 2012, 7 pages, 2012, Article ID 982683.

[16] S. A. Ramirez-Garcia, C. Charles-Niño, M. Mazariegos-Rubí et al., "Association of the ELMO1 gene (Snp rs1345365) with development of type 2 diabetes mellitus in the mexican mestizo population," Investigacion Clinica (Venezuela), vol. 56, no. 4, pp. 341-355, 2015.

[17] R. Gutiérrez-Vidal, A. Rodríguez-Trejo, S. Canizales-Quinteros et al., "LOC387761 polymorphism is associated with type 2 diabetes in the Mexican population," Genetic Testing and Molecular Biomarkers, vol. 15, no. 1-2, pp. 79-83, 2011.

[18] A. S. Jiménez-Osorio, S. González-Reyes, W. R. García-Niño et al., "Association of nuclear factor-erythroid 2-related factor 2 , thioredoxin interacting protein, and heme oxygenase-1 gene polymorphisms with diabetes and obesity in mexican patients," Oxidative Medicine and Cellular Longevity, vol. 2016, Article ID 7367641, 8 pages, 2016.

[19] A. I. Burguete-Garcia, M. Cruz-Lopez, V. Madrid-Marina et al., "Association of Gly972Arg polymorphism of IRS1 gene with type 2 diabetes mellitus in lean participants of a national health survey in Mexico: a candidate gene study," Metabolism: Clinical and Experimental, vol. 59, no. 1, pp. 38-45, 2010. 
[20] G. García-Elorriaga, M. Mendoza-Aguilar, G. del Rey-Pineda, and C. González-Bonilla, "Genetic polymorphisms of the tumor necrosis factor and lymphotoxin alpha in type 2 diabetes.," Revista Médica del Instituto Mexicano del Seguro Social, vol. 51, no. 1, pp. 42-49, 2013.

[21] E. A. Cameron, V. L. Martinez-Marignac, A. Chan et al., "MGEA5-14 polymorphism and type 2 diabetes in Mexico City," American Journal of Human Biology, vol. 19, no. 4, pp. 593-596, 2007.

[22] E. J. Parra, L. Cameron, L. Simmonds et al., "Association of TCF7L2 polymorphisms with type 2 diabetes in Mexico City," Clinical Genetics, vol. 71, no. 4, pp. 359-366, 2007.

[23] C. Maldonado-Bernal, O. A. Trejo-de la, M. E. SánchezContreras, N. Wacher-Rodarte, J. Torres, and M. Cruz, "Low frequency of Toll-like receptors 2 and 4 gene polymorphisms in Mexican patients and their association with type 2 diabetes," International Journal of Immunogenetics, vol. 38, no. 6, pp. 519523, 2011.

[24] E. Perez-Luque, J. M. Malacara, M. E. Garay-Sevilla, and M. E. Fajardo, "Association of the TNF- $\alpha-308 \mathrm{G} / \mathrm{A}$ polymorphism with family history of type 2 diabetes mellitus in a Mexican population," Clinical Biochemistry, vol. 45, no. 1-2, pp. 12-15, 2012.

[25] J. M. Guzmán-Flores, J. F. Muñoz-Valle, J. Sánchez-Corona et al., "Tumor necrosis factor-alpha gene promoter -308G/A and -238G/A polymorphisms in Mexican patients with type 2 diabetes mellitus," Disease Markers, vol. 30, no. 1, pp. 19-24, 2011.

[26] R. M. Cerda-Flores, R. A. Rivera-Prieto, B. Pereyra-Alférez et al., "Genetic structure of Mexican Mestizos with type 2 diabetes mellitus based on three STR loci," Gene, vol. 525, no. 1, pp. 41-46, 2013.

[27] V. L. Martinez-Marignac, A. Valladares, E. Cameron et al., "Admixture in Mexico City: Implications for admixture mapping of Type 2 diabetes genetic risk factors," Human Genetics, vol. 120 , no. 6 , pp. 807-819, 2007.

[28] Q. Ayub, L. Moutsianas, Y. Chen et al., "Revisiting the thrifty gene hypothesis via 65 loci associated with susceptibility to type 2 diabetes," American Journal of Human Genetics, vol. 94, no. 2, pp. 176-185, 2014.

[29] S. H. Kwak and K. S. Park, "Genetics of type 2 diabetes and potential clinical implications," Archives of Pharmacal Research, vol. 36, no. 2, pp. 167-177, 2013.

[30] E. Ahlqvist, T. S. Ahluwalia, and L. Groop, "Genetics of type 2 diabetes," Clinical Chemistry, vol. 57, no. 2, pp. 241-254, 2011.

[31] N. D. Palmer, C. W. McDonough, P. J. Hicks et al., "A genomewide association search for type 2 diabetes genes in african americans," PLoS ONE, vol. 7, no. 1, Article ID e29202, 2012.

[32] C. S. Janipalli, M. V. K. Kumar, D. G. Vinay et al., "Analysis of 32 common susceptibility genetic variants and their combined effect in predicting risk of Type 2 diabetes and related traits in Indians," Diabetic Medicine, vol. 29, no. 1, pp. 121-127, 2012.

[33] S. K. Das, "Genetic epidemiology of adult onset type 2 diabetes in Asian Indian population: past, present and future," International Journal of Human Genetics, vol. 6, no. 1, pp. 1-13, 2006.

[34] V. Lyssenko and M. Laakso, "Genetic screening for the risk of type 2 diabetes: worthless or valuable?" Diabetes Care, vol. 36, supplement 2, pp. S120-S126, 2013.

[35] A. M. Simonis-Bik, G. Nijpels, T. W. Van Haeften et al., "Gene variants in the novel type 2 diabetes loci CDC123/CAMK1D, THADA, ADAMTS9, BCL11A, and MTNR1B affect different aspects of pancreatic $\beta$-cell function," Diabetes, vol. 59, no. 1, pp. 293-301, 2010. 


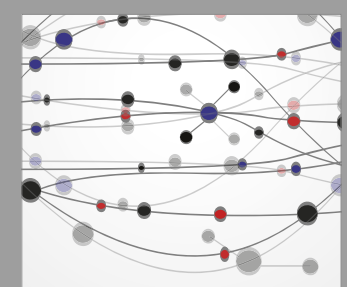

The Scientific World Journal
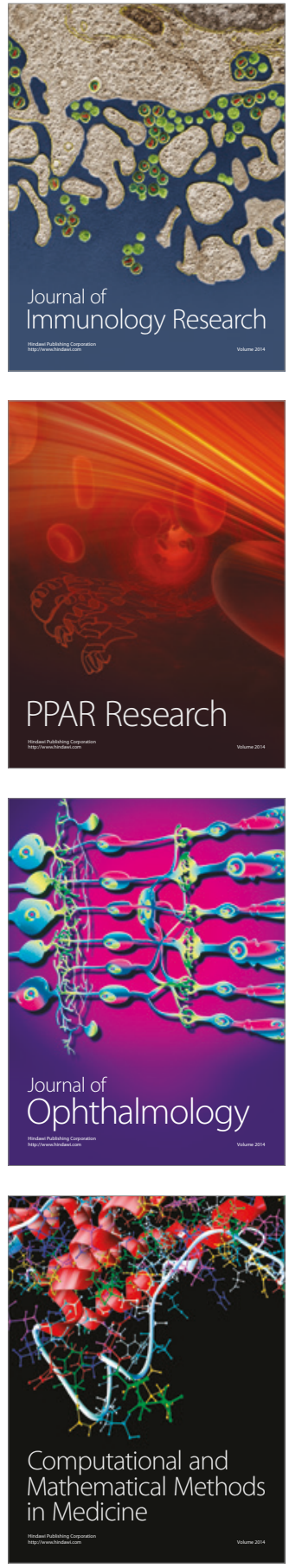

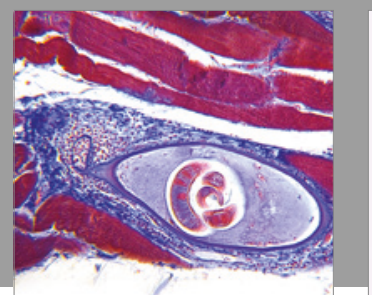

Gastroenterology Research and Practice
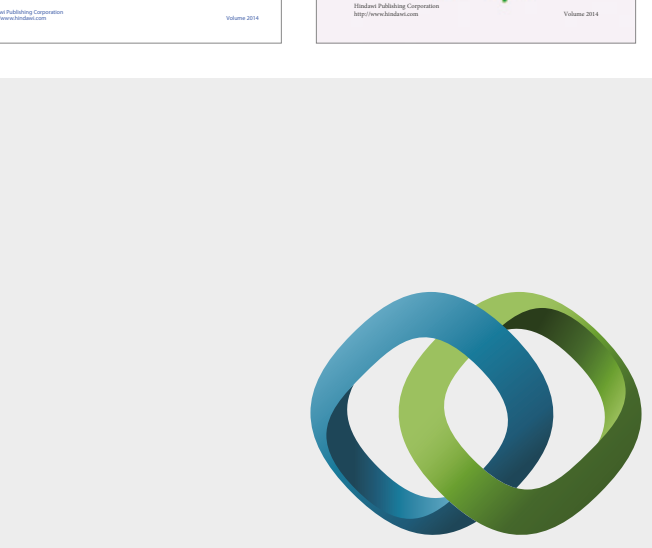

\section{Hindawi}

Submit your manuscripts at

https://www.hindawi.com
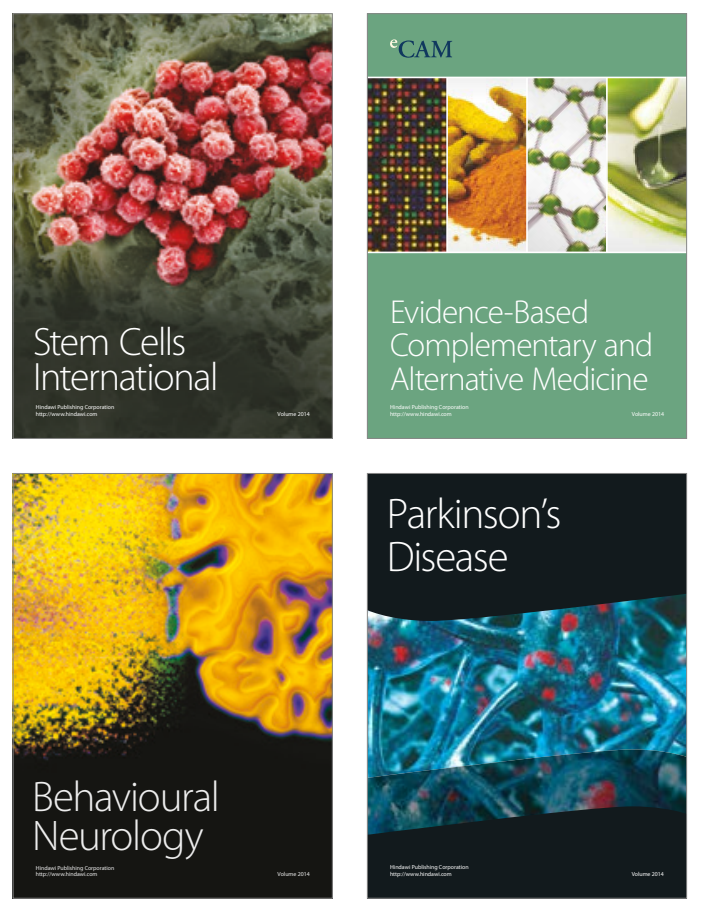
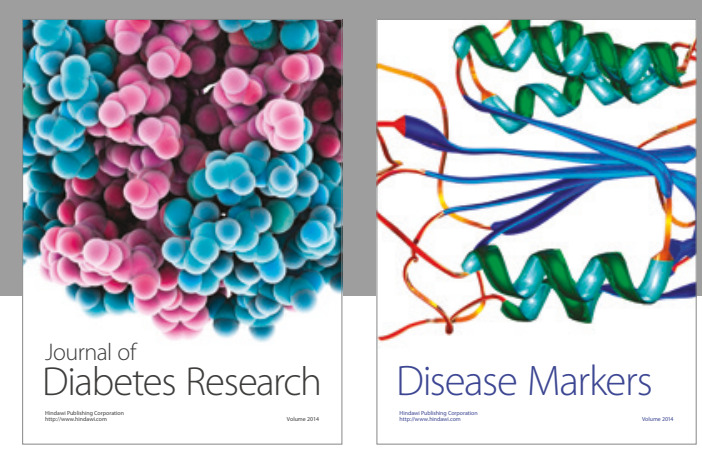

Disease Markers
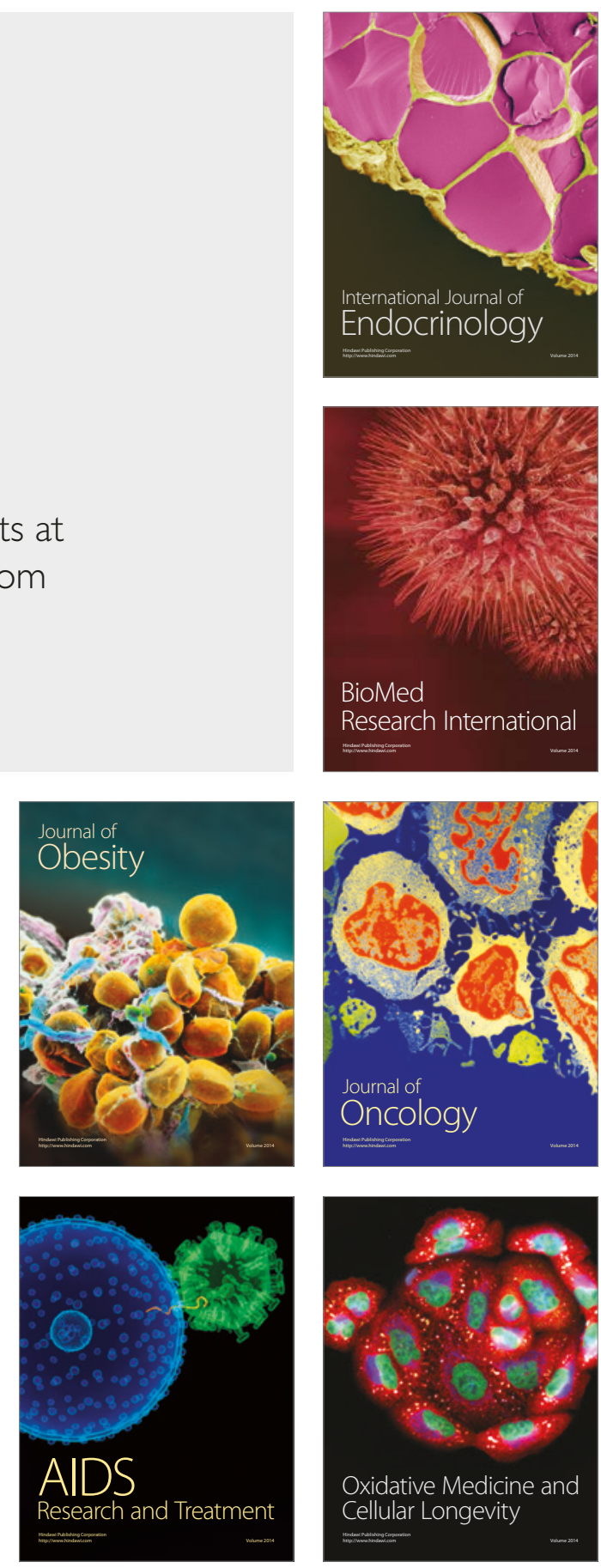